\title{
Fault Diagnosis for Machinery based on Feature Selection and Probabilistic Neural Network
}

\author{
HaipingLi*, Jianmin Zhao, Xinghui Zhang, Xianglong Ni \\ Mechanical Engineering College, Shijiazhuang, 050003, China
}

\begin{abstract}
Fault diagnosis for the maintenance of machinery is more difficult since it becomes more precise, automatic and efficient. To tackle this problem, a feature selection and probabilistic neural network-based method is presented in this paper. Firstly, feature parameters are extracted and selected after obtaining the raw signal. Then, the selected feature parameters are preprocessed according to the faulted characteristic frequencies of components. Finally, the diagnosis results are outputted with the decision method of PNN. Experimental data is utilized to demonstrate the effectiveness of this methodology.
\end{abstract}

Keywords: fault diagnosis; fault characteristic frequency; feature selection; probabilistic neural network

(Submitted on May 31, 2017; Revised on October 10, 2017; Accepted on October 18, 2017)

(C) 2017 Totem Publisher, Inc. All rights reserved.

\section{Introduction}

Mechanical equipment in modern industries becomes more precise, automatic and efficient. Therefore, researchers pay more attention to the fault diagnosis to fully inspect its health conditions. Diagnosing mechanical equipment faults usually has two techniques: data-driven methods and model-based methods. In model-based methods, the goal of signal models [2,3] is to find the characteristic frequencies of different faults (like bearing defect, gear failure or other breakdown). In practice, it is impossible to store all the vibration signals for vibration analysis. However, data-driven methods can diagnose the mechanical equipment faults using the features extracted from the vibration signals without the demand to store a large amount of data.

Su etc. [10] proposed an improved gear fault identification method based on Hilbert-Huang transform (HHT) to overcome the problem of reconstructing a feature matrix of singular value decomposition. In the paper, HHT technique was utilized to acquire instantaneous frequency and amplitude matrices from faulted gear signals. The adaptive variable steplength natural gradient blind source separation (BSS) algorithm was used in [1] to analyze the vibration signal for helicopter gearbox fault diagnosis. Lei and Zuo [7] proposed a new algorithm in classifying the different levels of gear cracks based on weighted $\mathrm{K}$ nearest neighbor. The algorithm made the characteristic frequency of faulty gears detect effectively. In addition, some other techniques were also utilized in this aspect, such as Hidden Markov Model (HMM) [5], support vector machine (SVM) [11], artificial neural network (ANN) [6] and so on.

As the equipment is becoming more and more complicated, sometimes the problem cannot be solved with only one technique. Hereby, the combination of two or three methods may be utilized. A fault detection method that combines Hilbert transform and wavelet packet transform was proposed to extract modulating signal and help detect the early gear fault [8]. Wu etc. [12] developed an intelligent diagnosis for gear fault identification and classification based on vibration signal using discrete wavelet transform and adaptive neuron-fuzzy inference system (ANFIS) to solve the problem of abnormal transient signals.

\footnotetext{
* Corresponding author.

E-mail address:hp_li@hotmail.com.
} 
However, determining the fault location with feature extraction is not very effective all the time because different features may be sensitive to different kinds of faults. Therefore, feature selection is needed after extracting features. Sometimes, only feature selection does not work perfectly. It is found in study that the diagnosis results are not very accurate when the faulted characteristic frequencies are close. In order to tackle this dilemma, a new fault diagnosis method based on feature selection and Probabilistic Neural Network (PNN) is proposed in this paper. PNN has more advantages, such as fast learning rate, low misjudgment rate, easy to use and so on. Therefore, it is very suited for pattern recognition, fault diagnosis and classification.

The remaining sections of this paper are organized as follows. Section 2 introduces the framework of the method proposed in this paper. In section 3, the experiment is described and the proposed method is applied to planetary gearbox fault diagnosis. Finally, section 4 gives the conclusions.

\section{Framework of the proposed method}

\subsection{Probabilistic neural network}

Probabilistic neural network (PNN) is one kind of artificial neural networks developed by Specht. D.F in 1988. It is a feedforward network derived from radial basis function (RBF) neural network. Compared with other neural networks, PNN is faster in convergence and higher in accuracy, which is suited to pattern classification and fault diagnosis [8,9]. PNN is a four-layer network which consists of input layer, pattern layer, summation layer and output layer, as shown in Figure 1.

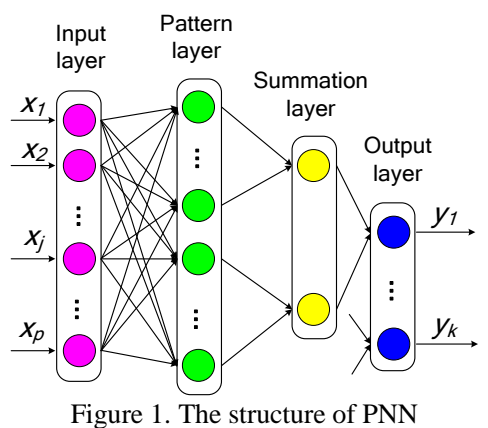

Input layer is not involved with calculation. Its function is to collect the training sample values. The dimensionality of the sample is the same as the neuron number. Namely, import Sample $X$ to the network.

The function of pattern layer is to get the correlativity between input sample and every pattern. The neuron number of the pattern layer is the same as the pattern number. The pattern layer distinguishes $X_{i j}$ to class $N$ by

$$
f_{i j}(\mathrm{x})=\frac{1}{(2 \pi)^{P / 2} \sigma^{p}} \exp \left[-\frac{\left(x-X_{i j}\right)^{T}\left(x-X_{i j}\right)}{2 \sigma^{2}}\right]
$$

where $m$ is the total number of pattern and $i=1,2, \ldots, m, j=1,2, \ldots, N_{i} . N_{i}$ is the sample number of the $i$ th pattern. $P$ is the number of space dimensionality of training sample. $\sigma \in(0, \infty)$ is the smoothing parameter. $X_{i j}$ is the $j$ th sample of the $i$ th pattern. $f_{i j}(x)$ is the $j$ th hidden layer central vector of the $i$ th pattern.

Summation layer averages all of the hidden layer central vectors to get the probability density estimation value of the $i$ th pattern, as follows.

$$
f_{i}(\mathrm{x})=\frac{1}{N_{i}} \sum_{j=1}^{N_{i}} f_{i j}(\mathrm{x})
$$

Each pattern has a specific summation unit, and the specific summation unit only connects with the neuron of its pattern. Then, add all the output results. 
The function of output layer is to select the maximum posterior probability density value $(f(x))$ from all probability density estimation values $\left(f_{1}(x), f_{2}(x), \ldots, f_{i}(x)\right)$. Then treat the $f(x)$ as the result of PNN and output it.

Assume there are $w$ kinds of fault modes $\theta_{1}, \theta_{2}, \ldots, \theta_{q}, \ldots, \theta_{w}$, for a test sample $x=\left(x_{1}, x_{2}, \ldots, x_{q}\right)^{\mathrm{T}}$, if

$$
h_{q} l_{q} f_{q}(x)>h_{k} l_{k} f_{k}(x), \quad k \neq q
$$

Then $x \in \theta_{q}$.

where $h_{q}, h_{k}$ are the prior probability of $\theta_{q}, \theta_{k} . l_{q}$ is cost factor that a sample belong to $\theta_{q}$ but judged to the other patterns. $l_{k}$ is cost factor that a sample belong to $\theta_{k}$ but judged to the other patterns. $f_{q}(x), f_{k}(x)$ are probability density estimation values of $\theta_{q}, \theta_{k}$.

$$
f_{q}(\mathrm{x})=\frac{1}{(2 \pi)^{P / 2} \sigma^{p}} \frac{1}{N_{q}} \sum_{i=1}^{N_{q}} \exp \left[-\frac{\left(x-X_{q j}\right)^{T}\left(x-X_{q j}\right)}{2 \sigma^{2}}\right]
$$

where $X_{q j}$ is the $j$ th sample of the $q$ th pattern. $N_{q}$ is the total number of pattern. $\sigma \in(0, \infty)$ is the smoothing parameter.

\subsection{The method proposed in this paper}

Having introduced the PNN and its constituting components, the procedure of applying the PNN based classification method for fault diagnosis is shown in Figure 2 and is summarized as follows:

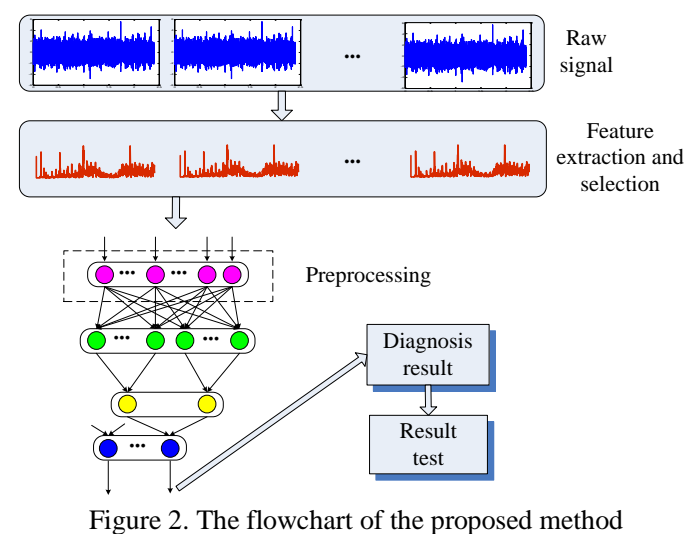

- Step 1. Collect the raw signal.

- $\quad$ Step 2. Extract and select feature parameters.

Extract 36 feature parameters from train data and test data; the 36 feature parameters can be seen in Table 1 . The equations and feature extraction methods of them can be seen in Ref. [4].

Different feature parameters may be sensitive to different kinds of faults. Therefore, feature selection is needed. This paper utilized the feature selection method proposed in Ref. [4]. The detailed introduction can be seen in Section 2.2.2. from Eq. (3) to (12).

- $\quad$ Step 3. Preprocess the selected feature parameters.

It is found in study that the diagnosis results are not very accurate when the faulted characteristic frequencies are close. For example, the faulted characteristic frequencies of planet gear and ring gear in a small planet gearbox are very similar so a preprocessing method is proposed in this paper. After achieving the selected feature parameters of different patterns, making feature parameters of one pattern divide by the faulted characteristic frequencies of another pattern, which the 
faulted characteristic frequencies of the two patterns are similar, is next. In other words, if faulted characteristic frequencies of two patterns, $f 1$ and $f 2$, are two close values, $F 1$ and $F 2$ are feature vectors of the two patterns, then $F 1 / f 2$ and $F 2 / f 1$ are the new feature vectors.

- Step 4. Output the diagnosis results according to the decision method of PNN.

- $\quad$ Step 5. Demonstrate the effectiveness of the method using test data.

\begin{tabular}{|c|c|c|c|c|c|}
\hline No. & Name & No. & Name & No. & Name \\
\hline$f_{l}$ & Maximum Value & $f_{2}$ & Minimum Value & $f_{3}$ & Peak to Peak \\
\hline$f_{4}$ & Average Value & $f_{5}$ & Mean Square Value & $f_{6}$ & RMS \\
\hline$f_{7}$ & Peak Value & $f_{8}$ & Variance & $f_{9}$ & Standard Deviation \\
\hline$f_{10}$ & Energy & $f_{11}$ & Skewness & $f_{12}$ & Kurtosis \\
\hline$f_{13}$ & Square Mean Absolute Root & $f_{14}$ & Mean Absolute Value & $f_{15}$ & Shape Factor \\
\hline$f_{16}$ & Crest Factor & $f_{17}$ & Impulse Factor & $f_{18}$ & Abundance Factor \\
\hline$f_{19}$ & Clearance Factor & $f_{20}$ & Mean Frequency & $f_{2 I}$ & Frequency Center \\
\hline$f_{22}$ & Root Mean Square Frequency & $f_{23}$ & Standard Deviation Frequency & $f_{24}$ & FM0 \\
\hline$f_{25}$ & FM4 & $f_{26}$ & FM4* & $f_{27}$ & M6A \\
\hline$f_{28}$ & M6A* & $f_{29}$ & M8A & $f_{30}$ & M8A* \\
\hline$f_{31}$ & NA4 & $f_{32}$ & NA4* & $f_{33}$ & NB4 \\
\hline$f_{34}$ & NB4* & $f_{35}$ & ER & $f_{36}$ & EOP \\
\hline
\end{tabular}

\section{A case study}

\subsection{Experimental setup and data acquisition}

A mechanical test rig in the RCM laboratory of Mechanical Engineering College is used in this research to validate the effectiveness of the proposed method in this paper. The structure of it can be seen in Figure 3 (1). The wear failure is seeded on one tooth of sun gear, planet gear and ring gear, respectively. The specific faults are shown in Figure 3 (2).

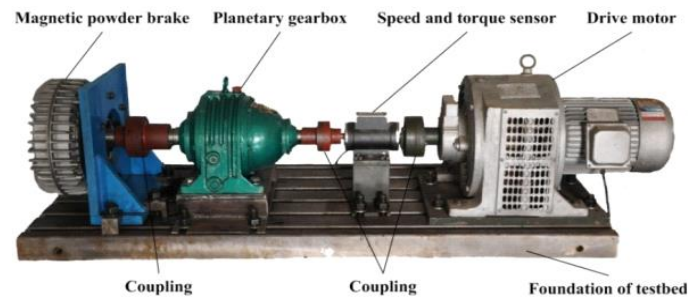

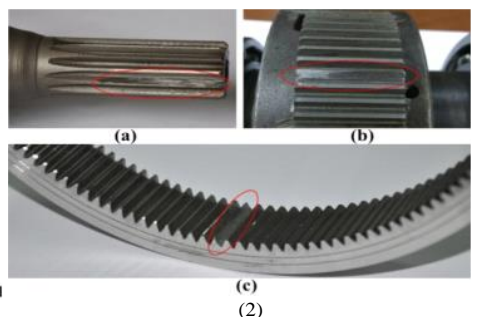

(2)

Figure 3. (1) Planetary gearbox test rig; (2) Seeded failure: (a) sun gear, (b) planet gear, (c) ring gear

The sampling frequency of this experimental system is $20 \mathrm{kHz}$. The experimental process can be seen in Ref. [4] and the configuration parameters of the planetary gearbox can be seen in Table 2 .

Table 2. Planetary gearbox configuration parameters
\begin{tabular}{|l|c|c|c|}
\hline Gear & Sun gear & Planet gear (number) & Ring gear \\
\hline Number of teeth & 13 & $64(3)$ & 146 \\
\hline
\end{tabular}

\subsection{Data analysis and discussion}

The raw signal acquired from the test rig when the rotary speed is $1200 \mathrm{rpm}$ is used to analyze. There are four pattern data, 1 normal state, 2 sun gear fault, 3 planet gear fault and 4 ring gear fault. In order to validate the effectiveness of the method proposed in this paper, six feature parameters are randomly selected. Three samples are also chosen as the train samples and the other 30 samples as the test samples, as Ref. [4] introduced. The diagnosis results can be seen in Figure 4 (a). It can be seen from the figure that the accuracy is only $25 \%$ when selecting six feature parameters randomly. Only the first pattern, normal state, is diagnosed exactly. 


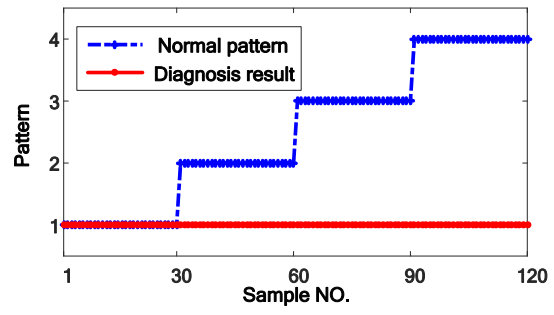

(a)

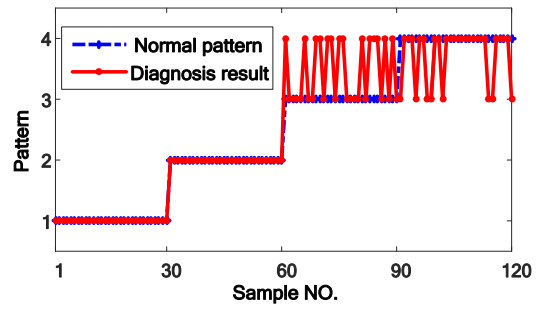

(b)

Figure 4. Diagnosis results: (a) select feature parameters randomly; (b) using the selected feature parameters

Figure 4(b) is the diagnosis results when selecting feature parameters using the method proposed in Ref. [4]. It can be seen from the figure that the accuracy is $81.67 \%$, which has been improved obviously. Though the samples of Pattern 1 and 2 are diagnosed exactly, some samples of Pattern 3 and 4 are misjudged easily. 22 samples of Pattern 3 and 4 are misjudged. Maybe the reason is that the faulted characteristic frequencies of planet gear and ring gear are close. Table 3 is the frequencies of planetary gearbox when the rotary speed is $1200 \mathrm{rpm}$.

Table 3. Faulted characteristic frequencies of every gear

\begin{tabular}{|c|c|c|c|c|}
\hline $\begin{array}{l}\text { Rotary speed } \\
(\mathrm{rpm})\end{array}$ & $\begin{array}{l}\text { Meshing frequency } \\
(\mathrm{Hz})\end{array}$ & $\begin{array}{l}\text { Fault frequency of } \\
\text { sun gear }(\mathrm{Hz})\end{array}$ & $\begin{array}{l}\text { Fault frequency of } \\
\text { planet gear }(\mathrm{Hz})\end{array}$ & $\begin{array}{l}\text { Fault frequency of ring } \\
\text { gear }(\mathrm{Hz})\end{array}$ \\
\hline 1200 & 238.738 & 55.093 & 3.730 & 4.906 \\
\hline
\end{tabular}

Figure 5 (a) is the diagnosis results using the method proposed in this paper. The selected feature parameters are preprocessed according to the faulted characteristic frequencies of planet gear and ring gear. Assume $f_{p}$ and $f_{r}$ are the faulted characteristic frequencies of planet gear and ring gear, and $F_{p}$ and $F_{r}$ are feature vectors of planet gear failure and ring gear failure pattern, which means $F_{p} / f_{r}$ and $F_{r} / f_{p}$ are the new feature vectors. It can be seen from the figure that the accuracy of the method proposed in this paper is $100 \%$. Therefore, the method is effective.

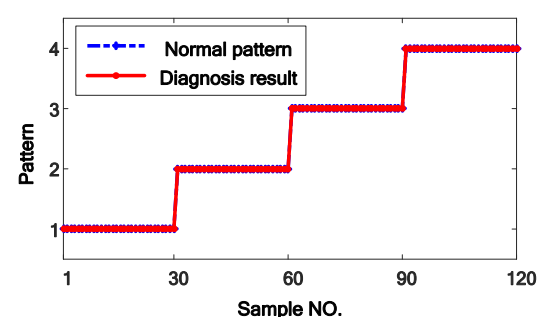

(a)

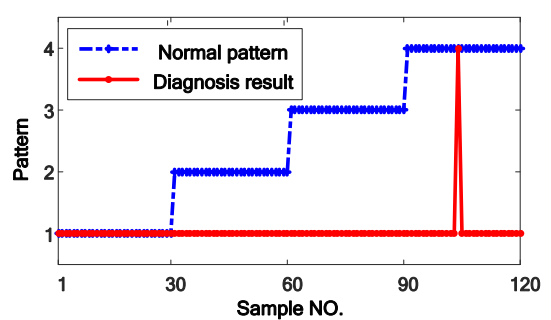

(b)

Figure 5. Diagnosis results: (a) using the method proposed in this paper;(b) using random feature parameters and signal preprocessing

In order to validate the effectiveness of the method proposed in this paper further, the random feature parameters are utilized. And the random feature parameters are preprocessed according to the faulted characteristic frequencies of planet gear and ring gear as before. The diagnosis results can be seen in Figure 5 (b). It can be seen from the figure that the signal preprocessing does not work. The accuracy improved only a little. Therefore, feature selection is important.

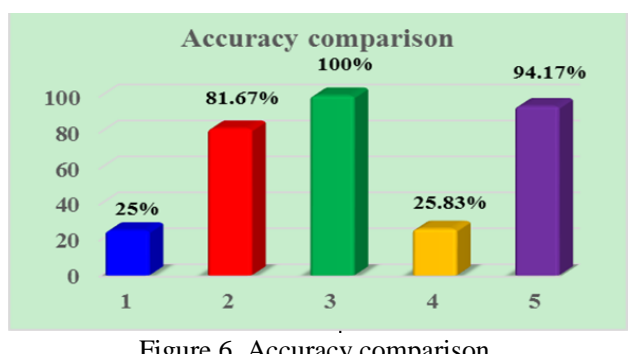

Figure 6 is the accuracy comparison of the method introduced above and EMD-EDT method proposed in Ref. [4]. The first is the diagnosis results when select feature parameters randomly; the second is the diagnosis results when select feature parameters using the method proposed in Ref. [4]; the third is the diagnosis results using the method proposed in this paper; the fourth is the diagnosis results using random feature parameters and signal preprocessing; the fifth is the diagnosis results using the EMD-EDT method proposed in Ref. [4]. The effectiveness of the method proposed in this paper can be demonstrated through the comparison. 


\section{Conclusions}

In this paper, a novel method based on feature selection and Probabilistic Neural Network (PNN) is proposed to tackle the problem that fault diagnosis for the maintenance of machinery is more difficult with it becoming more precise, automatic and efficient. In the proposed method, feature parameters are extracted and selected after obtaining the raw signal. Then, the selected feature parameters are preprocessed according to the faulted characteristic frequencies. Finally, the diagnosis results are outputted with the decision method of PNN. A planetary gearbox test rig is established as example and four kinds of states are utilized. The effectiveness of the proposed method is validated by the experimental data.

\section{References}

1. Y. Chen, "Study on BSS Algorithm used on Fault Diagnosis of Gearbox," TELKOMNIKA Indonesian Journal of Electrical Engineering, vol. 11, no. 6, pp. 2942-2947, 2013.

2. A. K. Jalan and A. R. Mohanty, "Model based fault diagnosis in rotating machinery," International Journal of Performability Engineering, vol. 7, no. 6, pp. 515-523, 2011.

3. Y. H. Jiang, B. P. Tang, Y. Qin and W. Y. Liu, "Feature extraction method of wind turbine based on adaptive Morlet wavelet and SVD," Renewable Energy, vol. 36, no. 8, pp. 2146-2153, 2011.

4. H. P. Li, J. M. Zhao, J. Liu and X. L. Ni, "Application of Empirical Mode Decomposition and Euclidean Distance Technique for Feature Selection and Fault Diagnosis of Planetary Gearbox," Journal of Vibroengineering, vol. 18, no. 8, pp. 5096-5113, 2016.

5. J. S. Kang and X. H. Zhang, "Application of Hidden Markov Models in machine fault diagnosis," Information-An International Interdisciplinary Journal, vol. 15, no. 12B, pp. 5829-5838, 2012.

6. K. Kumar, "Knowledge Extraction From Trained Neural Network," TELKOMNIKA Indonesian Journal of Electrical Engineering, vol. 1, no. 4, pp. 282-293, 2012.

7. Y. G. Lei and M. J. Zuo, "Gear crack level identification based on weighted K nearest neighbor classification algorithm," Mechanical Systems and Signal Processing, vol. 23, no. 5, pp. 1535-1547, 2009.

8. S. R. Mohanty, P.K. Ray and N. Kishor, "Classification of disturbances in hybrid DG system using modular PNN and SVM," ELSEVIER Electrical Power and Energy Systems, vol. 44, pp. 764-777, 2013.

9. D. H. Qiu, Y. Chen and Y. Li, “Adaptive RBF neural networks sliding mode control for a DEAP linear actuator," International Journal of Performability Engineering, vol. 13, no. 4, pp. 400-408, July 2017.

10. Z. Y. Su, Y. M. Zhang, M. P. Jia, F. Y. Xu and J. Z. Hu, "Gear fault identification and classification of singular value decomposition based on Hilbert-Huang transform," Mechanical Science and Technology, vol. 25, no. 2, pp. 267-272, 2011.

11. X. L. Tang, L. Zhuang, J. Cai and C. B. Li, "Multi-fault classification based on support vector machine trained by chaos particle swarm optimization," Knowledge-Based Systems, vol. 23, pp. 486-490, 2010.

12. J. D. Wu, "Fault gear identification and classification using discrete wavelet transform and adaptive neuron-fuzzy inference," Expert Systems with Applications, vol. 36, no. 3, pp. 6244-6255, 2009.

Haiping Li received the B.S. degree from Beijing Institute of Technology, Beijing, China, in 2012; M.S. degree from Mechanical Engineering College, Shijiazhuang, China, in 2014. He is now a Ph.D. student studying in Mechanical Engineering College, Shijiazhuang, China. His main research interests include digital signal processing, mechanical fault diagnosis and fault prognosis.

Jianmin Zhao received the B.S. and M.S. degree from Tsinghua University, Beijing, China, in 1985 and 1988 , respectively. He received Ph.D. degree from the University of Birmingham, Birmingham, UK, in 2007. He is currently a professor at Mechanical Engineering College, Shijiazhuang, China. His main research interests include degradation modeling for mechanical components, maintenance decision making and warranty return policy.

Xinghui Zhang received the B.S., M.S. and Ph.D. degree from Mechanical Engineering College, Shijiazhuang, China, in 2005, 2010 and 2015, respectively. His main research interests include mechanical fault diagnosis, fault prognosis, performance based contracts and digital signal processing.

Xianglong Ni received the B.S. degree from Jimei University, Xiamen, China, in 2011; M.S. degree from Mechanical Engineering College, Shijiazhuang, China, in 2013. He is now a Ph.D. student studying in Mechanical Engineering College, Shijiazhuang, China. His main research interests include digital signal processing, degradation modeling for mechanical components and maintenance decision making. 